\title{
Telephony over Metropolitan Area Ad Hoc Networks: From Concept to Field Test
}

\author{
Eduard Glatz \\ Computer Engineering and \\ Networks Laboratory \\ ETH Zurich, Switzerland \\ Email: eglatz@tik.ee.ethz.ch
}

\author{
Ulrich Fiedler \\ Bern University of Applied Sciences \\ Engineering and Information Technology \\ Biel/Bienne, Switzerland \\ Email: ulrich.fiedler@bfh.ch
}

\begin{abstract}
Self-organizing multi-radio mesh networks can be used to establish metropolitan ad hoc telephony networks in disaster recovery situations and public safety settings such as after a flood or a terrorist attack. These networks can be employed to enable forces to extend the remaining circuitswitched communication infrastructure. This paper introduces a system architecture, a self-organized topology construction and channel allocation algorithm and its implementation in a distributed protocol. The architecture relies on commercially available IEEE 802.16-2004 (WiMAX) equipment that can be employed to build routers and gateways to a wired network. The topology construction and maintenance are based on a modified Dijkstra shortest path algorithm that maximizes a combined metric of path qualities and gateway load. Path qualities are derived from link qualities determined by SINR measurements. The channel allocation greedily allocates the locally least-interfered channel. The protocol includes four subprotocols: a join protocol, a hello protocol, a leave protocol, and a conflict resolution protocol to resolve inconsistencies in topology data across the network. The proposed architecture, algorithms, and protocol have been assessed via extensive simulations and implemented on hardware. Both, simulation results and field tests confirm their applicability/usability. ${ }^{1}$
\end{abstract}

Index Terms-Ad Hoc Networks; Topology Construction; Circuit Emulation; IEEE 802.16;

\section{INTRODUCTION}

In many disaster recovery scenarios, e.g., when recovering from terrorist attacks, voice communication is of paramount importance [1]. In such scenarios, it may not be possible or appropriate for the forces engaged in recovery operations to use parts or all of the existing infrastructure. Thus, extending the remaining telephone network infrastructure with a selfconfiguring multi-radio ad hoc network is attractive. Each router of this ad hoc network can then open up a service domain that enables mobile telephony for forces. The network can cover a metropolitan area of about $10 \mathrm{~km}^{2}$. The service domains may overlap, but can also be isolated. Much research has been carried out to optimize dedicated problems in ad hoc networks such as topology construction and channel allocation to maximize capacity [2], [3], [4]. However, to our knowledge little effort has been made to combine algorithms and protocols in a way that leads towards deployment.

\footnotetext{
${ }^{1}$ This research is supported by the Hasler Foundation.
}

This paper introduces an IEEE 802.16-2004 (WiMAX) based system architecture, a self-organized topology construction and channel allocation algorithm and its implementation in a distributed protocol. The proposed architecture is geared towards employing IEEE 802.16's support for quality of service to be able to connect the standard telephone equipment that forces are used to. The topology construction and maintenance are based on a modified Dijkstra shortest path algorithm that trades path qualities and the load on gateways. Path qualities are derived from link qualities determined by SINR (Signal and Interference to Noise Ratio) measurements. Thus, interference caused by external RF sources is included in link quality estimates. Furthermore, self-interference between routers participating in the network is handeled by a channel allocation that greedily allocates the locally least-interfered channel. We then propose a protocol for a distributed implementation of the topology construction and maintenance including channel allocation. This implementation assumes that each router maintains its own topology database with link states. The protocol employs unacknowledged optimistic flooding based on IEEE 802.16 cell broadcasts combined with a mechanism to resolve conflicts in topology databases due to lost link state messages. The protocol consists of four subprotocols: The conflict resolution protocol (CRP) to resolve conflicts in the topology databases of neigboring routers that come from message loss, the join protocol (JP) that joins new routers to the network, the hello protocol to maintain neighbor relationships, and the leave protocol to handle gracefully router leaves. The usability of this architecture, algorithm, and protocol has been proven by implementing it on hardware which has been evaluated in a field-test.

The structure of the rest of this paper is organized as follows: Section II introduces the system architecture. Section III reviews the topology construction algorithm. Section IV describes the protocol. Section V summarizes the evaluation results. Section VI discusses its performance. Section VII reviews the tests. In section VIII we conclude.

\section{Architecture}

The architecture we propose aims at employing commercially available IEEE 802.16-2004 equipment for telephone circuit emulation over the ad hoc network. This technol- 


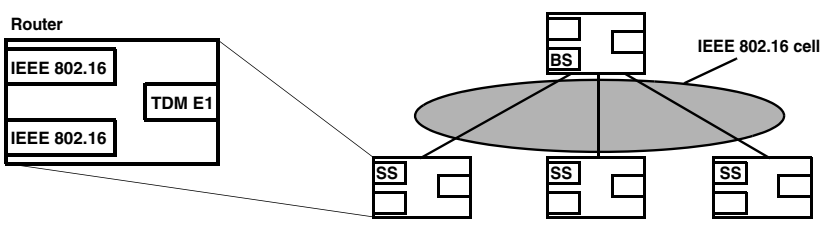

Fig. 1. An IEEE 802.16 cell connecting four wireless routers. The router on top acts as base station (BS) by opening up a cell serving three subscriber stations (SS) shown on the bottom. Each router contains a TDM E1 interface to connect circuit-switched telephone equipment.

ogy is of particular interest in these scenarios since IEEE 802.16 significantly differs from widely deployed IEEE 802.11 technology in terms of quality of service due to the use of TDMA compared to CSMA/CA. TDMA as defined by IEEE 802.16 implements cells connecting a base station (BS) with a set of subscriber stations (SS) as shown in fig. 1. Furthermore, the base station interface manages the quality of service of the subscriber station routers in its cell and offers a cell broadcast service for sending messages to all subscriber stations in the cell. Outages that last longer than $25 \mathrm{~ms}$ result in cutting off the telephone circuit emulation [10] [11] and cause telecommunication equipment such as GSM/UMTS base stations to reboot. Thus, constructing a full mesh topology and performing re-routing on top of this topology in case of a link failure is not superior to reordering the topology of a spanning tree. Moreover, a spanning tree suffers less from QoS degradation due to interference from spatial frequency reuse. Thus, we propose to employ routers with two wireless interfaces, one in BS mode and one in SS mode and construct a spanning tree topology as depicted in fig. 1. To enhance capacity gateways (GW) to the wired network have their two wireless interfaces both configured to BS mode. Moreover, we introduce a master gateway (MG) which has a wired connection to all other gateways and is the root of the spanning tree.

\section{ALGORITHM}

Our algorithm for dual topology construction and channel allocation is based on a modified Dijkstra shortest path algorithm for topology construction and on a greedy allocation of the locally least-interfered channel [6]. The modified Dijkstra algorithm maximizes path qualities instead of minimizing paths. Illustratively explained, instead of relaxing springs, the modified Dijkstra makes a move like a wavefront to join one router after another to the network. The wavefront starts at the master gateway (MG, 1 in fig. 2) and first invites all gateways ( 2 and 3 ) as children of the MG. Then, all remaining routers are joined one after another in a sequence defined by the Dijkstra algorithm. In fig. 2 a situation is shown where routers 1 to 6 are part of the network and routers 7 and 8 are join candidates. Next, these routers in front of the wavefront are invited to join the network in a way that maximizes path qualities from join candidate routers to the gateways while balancing the load on the base station interfaces of the gateways. The trade-off between path quality $p q$ and gateway interface load $l m$ is achieved with a composite path metric

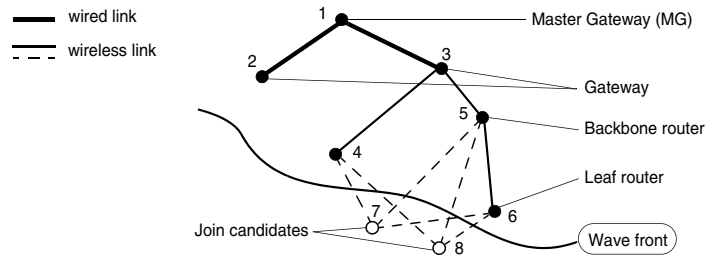

Fig. 2. The next router to join is chosen among the join candidates waiting outside of the wavefront. Upon join, the wavefront is extended to include the new routers.

cpm where $w$ is the balancing factor.

$$
c p m=w * p q+(1-w) * l m, 0 \leq w \leq 1 .
$$

The channel frequency allocation traverses the list of all available frequencies and allocates the least-interfered channel to minimize self-interference across the network. This channel allocation is locally optimal and achieves an approximation to the global minimum [7]. For a detailed description of the algorithm refer to [6].

\section{IMPLEMENTATION}

Next we introduce a protocol for distributed implementation of the topology construction and maintenance algorithm including channel allocation [9]. This protocol assumes that each router in the network keeps its own topology database (TDB), which conceptually is comparable to the link state database of OSPF [12]. The TDB contains link state advertisements (LSAs) of type ADD (a router has joined the network) and DEL (a router has been disconnected or the link is dead). The LSAs are issued by the parent router managing the cell where ADD or DEL occurred.

Databases of neighboring routers may be inconsistent due to loss of link advertisement messages. To synchronize these databases we define a conflict resolution protocol (CRP) that employs unacknowledged optimistic flooding of hashes of the database based on IEEE 802.16 cell broadcasts to detect inconsistencies.

Join-candidate routers first associate with a temporary parent to disseminate their neighborhood scan results in the network. Each temporary parent runs the topology construction algorithm after a waiting period to elect the next joiner and determine the joiner's permanent parent. Then the temporary parent informs the joiners which disassociate and reassociate if the permanent parent is not equal to the temporary parent.

Moreover, we implement a hello protocol to maintain neighbor relationships, and a leave protocol to handle router leaves.

\section{A. The Join Protocol}

The interactions of the join process are depicted in fig. 3 . At start a join-candidate router performs a scan to estimate the quality of potential links from itself to all routers in range. Then it associates with the router on the link with the best link quality. Through this join, this router becomes the temporary parent of the join candidate. The temporary parent accepts the Invite Request that includes a scan table containing all potential links from the join candidate to routers 


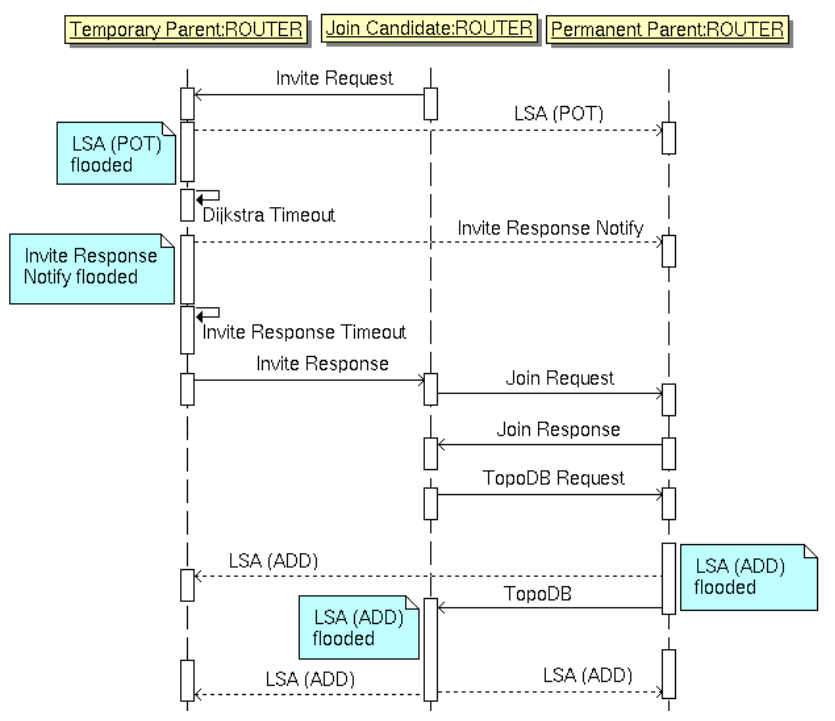

Fig. 3. This sequence diagram depicts the process of a new router joining the network. During a waiting period, the temporary parent gathers invite requests from join candidate routers which are immediately flooded through the network. Then after a timeout a join decision is taken and announced to the candidate. The candidate may then join.

in the network and floods this request throughout the network. Then the temporary parent waits for a fixed period of time (Dijkstra Timeout) to allow further join candidates to send Invite Requests. Then one of the join candidates is determined by the Dijkstra topology construction algorithm to definitively join the network. The Dijkstra Timeout allows a trade off between topology optimality and speed in topology construction. After this timeout the temporary parent runs the topology construction algorithm on the local topology database and all Invite Requests received during the wait period. The join decision is then announced to all join candidates via an Invite Response. This Invite Response is also flooded throughout the network to inform all routers that a join process has started. All routers having received a Invite Response start a timer to prevent concurrent joins. If the permanent parent of the elected join candidate is not equal to the temporary parent, which is rarely the case (see fig. 6), the candidate disassociates from the temporary parent and reassociates with the permanent parent. Then the join candidate exchanges a Join Request and Join Response before the topology database is exchanged and floods an $L S A$ of type ADD throughout the network. This $L S A(A D D)$ commits the join process. Lost messages are retransmitted twice to enable completion of the join process under adverse network conditions. Moreover, lost LSAs are reproduced by the conflict resolution.

\section{B. Conflict Resolution Protocol}

The CRP works as depicted in fig. 4. Each parent router managing a cell broadcasts the hash value of its topology database (TDB) to its child routers. This topoDB hash permits to check the equality of two TDBs. The child applies the hash function to its TDB and checks whether the received hash values corresponds to the computed hash values. If the hash

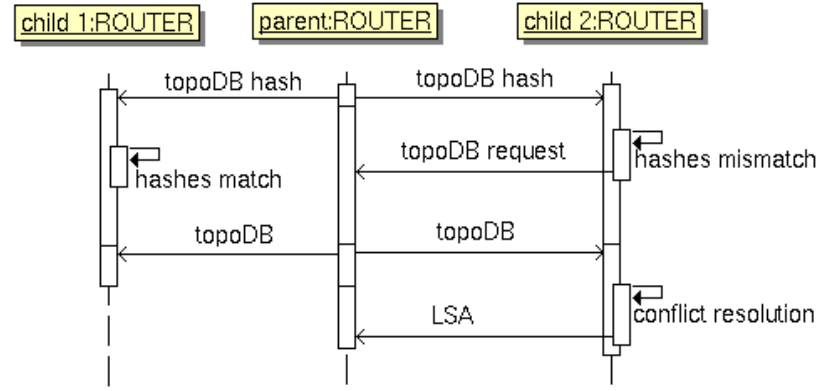

Fig. 4. This sequence diagram depicts the process of conflict resolution between a child and a parent router. The parent periodically sends a cell broadcast with a hash of its topology database. All children compare this hash against the hash of their local topology database and request the parent's database upon mismatch to resolve the conflict.

values are unequal, the child router solicits the corresponding TDB from its parent (sends a topoDB request to the parent). Hash collisions are dissolved by including a random number into the hash. As response, the parent cell broadcasts its TDB via topoDB to its child routers. When the child router receives this TDB, it resolves all conflicts between this TDB and its local copy before it sends back those LSAs that are missing in the received topoDB back. The current implementation of the CRP detects whether a link is in one TDB but not in the other TDB, or if the link is in both TDBs but with different sequence numbers. This conflict resolution between two links is performed solely based on sequence numbers, i.e., on a cell level, and the link entry with the higher sequence number wins.

\section{The Hello and Leave Protocol}

In addition the protocol has a hello sub-protocol to detect dead links and a leave sub-protocol for graceful leaves.

\section{Simulation Results}

We have implemented the proposed topology construction, maintenance and channel allocation algorithms in the QualNet simulator [5] using its IEEE 802.16-2005 compliant physical layer model. Our simulations focus on the evaluation of two aspects of the overall system architecture: the performance of the topology construction algorithm and the way this is affected by the topology maintenance procedure. In line with the application scenario requirements we simulated telephony circuit-switched traffic as a pair of 2 Mbps constant bit rate (CBR) streams between each mesh router and a gateway (see [10] and [11] for details). Furthermore, we applied the physical layer settings preferred by our industrial partner $(25 \mathrm{~mW}$ transmission power, 12 orthogonal channels of $10 \mathrm{MHz}$ width of the $5.8 \mathrm{GHz}$ band).

We summarize the results of the simulations in the following sections. For a more comprehensive review of the evaluation results, refer to [8].

\section{A. Topology Construction}

We investigated (i) the coverage, (ii) the selection of path metrics and (iii) the trade off between path quality and gateway 


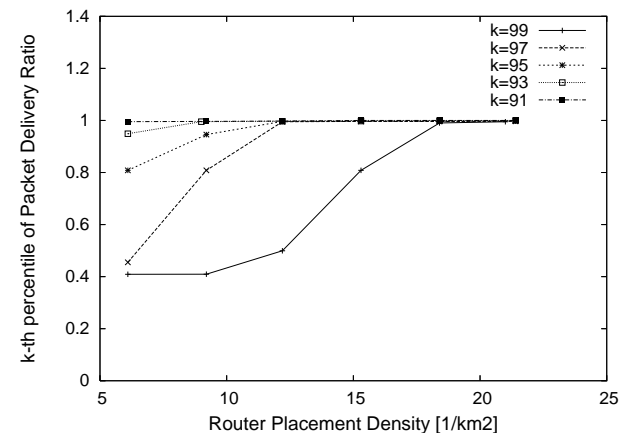

Fig. 5. The figure shows the minimum packet delivery ratio obtained for $\mathrm{k} \%$ $(\mathrm{k}=91,93,95,97,99)$ of the links. A reasonable PDR can only be achieved when more than 12 routers are placed per square kilometer.

load. Reviewing the results of our simulations, we find that the density of the router placement in the scenario has to be above 12 per square kilometer (see fig. 5). Respecting this constraint we note that the resulting packet delivery ratio, maximum delay and jitter ensure a performance within the limits set by telephony standards [10] [11] for at least $97 \%$ of the links. Investigating how the choice of path quality metric impacts performance, we find that this choice has little impact on the packet delivery ratio. In terms of SINR, a path quality metric based on a step function yields the best performance. This finding can be explained by the fact that a step function is the most appropriate approximation to model the SINR to link capacity relationship (for details refer to [6]). Next, we investigate the trade off between path quality and gateway load. The simulation results show that accounting for gateway load significantly improves the packet delivery ratio. We note that trading path qualities and traffic load on gateways to the wired network is a prerequisite to meet the stringent quality of service requirements of telephony circuit emulation. However, the details of the choice of the trade-off parameter $w$ in equation 1 is not significant as long as $w$ is between 0.6 and 0.9 [6].

\section{B. Topology Maintenance}

We investigate the impact of topology maintenance on the network performance once the initial topology has been constructed. Firstly, we analyze the behavior in a situation where randomly selected routers leave the network. Secondly, we evaluate the performance when new routers join the network.

1) Leaves of Routers: Analyzing the properties of TOWN topologies, we find that they are very flat, i.e. the fraction of backbone routers is low. As a result, the path length between a router and an associated gateway is typically short (see fig. 6 left-hand side). As a consequence, disconnecting a backbone router does not leave many dependent routers disconnected. In addition, simulations show that leaves do not deterioriate the network performance significantly.

2) Joins of Routers: We observe that subsequent joins of routers do decrease the fraction of backbone router. As a result, the fraction of backbone routers drops to approximately $7 \%$ and stabilizes at that level (see fig. 6 right-hand side). Then we
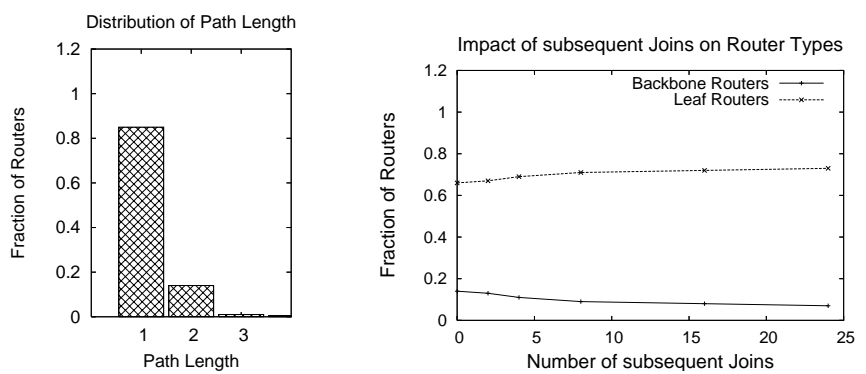

Fig. 6. The figure on the left side shows that the path length between a router and its associated gateway is typically short. This short path length can be explained with the fact that a gateway can serve four routers on average. The figure on the right shows that the fraction of backbone routers decreases for subsequent joins.

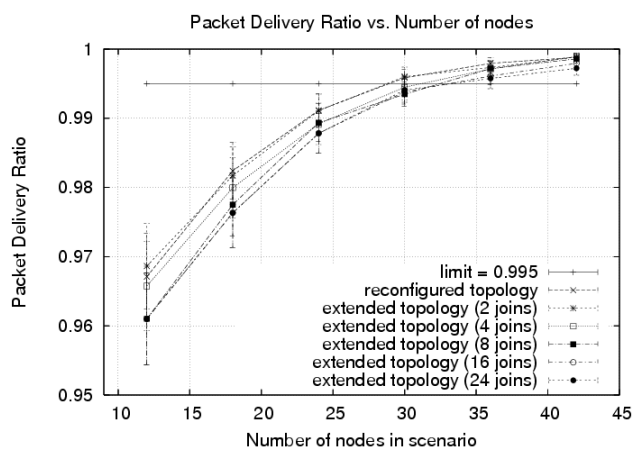

Fig. 7. The packet delivery ratio of an initial (reconfigured) topology is compared with the results obtained for networks that have been extended by joining routers. The recommended router density of $12 / \mathrm{km}^{2}$ equals 24 routers in the investigated scenario size of $1400 \mathrm{~m} \mathrm{x} 1400 \mathrm{~m}$.

compare the performance of an initial topology with network topologies that have been modified by subsequent joins of new routers. We find that the decrease of the packet delivery ratio for extended networks, while significant, is not critical for the intended application as long as the recommended router density is respected (see fig. 7). As a consequence, a tight setting of the Dijkstra Timeout (see IV-A) can be applied without major impact on overall performance.

\section{Performance Analysis}

We derive lower and upper bounds from the protocol definition to frame the duration of the topology construction. Both bounds are determined by timeout values used in the protocol state machine and the total count $n$ of routers to be joined. The lower bound includes all wait times and thus is given by

$$
t_{l b}=(T 1+T 2) * n
$$

with the following parameters:

\section{T1: Dijkstra Timeout}

T2: Invite Response timeout

The Dijkstra timeout is a trade off between topology optimality and speed in topology construction. The Invite Response time forces the temporary parent to wait until the LSA flooding is complete. 
The upper bound equals the maximum time for a join process not experiencing any timeout. It can be calculated as follows

$$
t_{u b}=(T 3+T 4+T 5+T 6) * n
$$

with the following parameters:

T3: association timeout

T4: Invite Response timeout

T5: Join Response timeout

T6: topoDB timeout

The choice of appropriate values for timeouts is influenced e.g., by the expected propagation times, message losses and technology-dependent properties like channel switching times. Optimized parameter settings will be identified during planned tests with final hardware.

\section{TESTS}

\section{A. In the Virtual Test Environment}

The protocol has been developed and tested in a virtual test environment that runs under VMware on a single high-end PC. The protocol resides in the management unit of each virtual router and employs the same interface for communication as on real hardware. However, underneath the interface is a network emulation which abstracts the wireless network. This network emulation works on a per protocol message basis and allows the engineer to test the protocol with configurable message delay and loss for each message type. Thus, functional tests under various network conditions (loss and delay rates, message type specific loss and delay rates) indicated that our protocol is correct before we ported it onto real hardware.

\section{B. Preliminary Field Test}

To be able to conduct a preliminary field test with hardware readily available we employed off-the-shelf IBM R50 laptops, and equipped them with 3Com IEEE 802.11 cards in addition to their internal Intel PRO card. We first started with indoor tests before we went outdoor since the indoor transmission range is smaller and the building's network facilitates centralizing log files for debugging and updating test configurations. First results can be summarized as follows: Under good network conditions, a router can join the network in several seconds. The join time is more or less constant with the number of routers and increases with the depth of the spanning tree network topology. All measured join times are above the theoretical limit. With six routers, the maximal join time measured with the timer configuration preferred by our industry partner was approximately 30 seconds. Churn, i.e., reconnects did not increase these times.

More comprehensive tests are needed, and thus are currently in progress using real WiMAX hardware.

\section{CONCLUSION AND FURTHER WORK}

In this paper we introduce a system architecture, a selforganized topology construction and channel allocation algorithm and its implementation in a distributed protocol. The architecture, algorithm and the protocol are tailored but not limited to telephony use over IEEE 802.16 equipment on a metropolitan scale. The topology construction and maintenance are based on a Dijkstra best path algorithm trading path qualities and gateway load. The link qualities to compute path qualities are derived from SINR measurements. The channel allocation greedily allocates the locally leastinterfered channel. The protocol makes use of a topology database of the entire network stored by each router, and relies on optimistic flooding that can be efficiently implemented with cell broadcasts. The protocol includes four sub-protocols: The join protocol for attaching new routers to the network, the conflict resolution protocol for resolving conflicts in the topology databases of neigboring routers that come from message loss, the hello protocol to maintain neighbor relationships, and the leave protocol to handle router leaves. The usability of both algorithm and protocol has been proven by implementation on hardware, which has been evaluated with positive results in a field-test.

As next steps we intend to refine our protocol to support more sophisticated techniques of both up- and downstream SINR measurements as well as background scans with a third interface.

\section{ACKNOWLEDGMENT}

We thank our industry partner Ascom (Schweiz) AG, especially Jörg Furrer.

\section{REFERENCES}

[1] M. Masellis, M.M. Ferrara, and S.W.A. Gunn, Fire Disaster and Burn Disaster: Planning and Management. Annals of Burns and Fire Disasters, vol. XII, June 1999.

[2] I.F. Akyildiz and X. Wang, A Survey on Wireless Mesh Networks. IEEE Radio Communications, September 2005.

[3] $\mathrm{H}$. Ju and I. Rubin, Mesh Topology Construction for Interconnected Wireless LANs. SECON05, 2005.

[4] A. Raniwala and T. Chiueh, Architecture and Algorithms for an IEEE 802.11-Based Multi-Channel Wireless Mesh Network. Proc. of Infocom, March 05.

[5] Scalable Network Technologies, Inc., QualNet 4.0 Network Simulator. http://www.qualnet.com/

[6] U. Fiedler and E. Glatz, Self-organized Topology Construction and Channel Allocation for Multi-IEEE 802.16-2004 Radio Routers in Disaster Recovery. Proc. 1st workshop on WiMAX, wireless, and mobility (WEIRD07), Coimbra, Portugal, 2007. Available: http://www.tik.ethz.ch (see section: Publications)

[7] U. Fiedler, Mesh Network Topology Construction and Channel Frequency Allocation in the TOWN Project. TIK-report No. 255, Computer Engineering and Networks Laboratory, ETH Zurich, Sept. 2006. Available: http://www.tik.ethz.ch (see section: Publications)

[8] U. Fiedler and E. Glatz, Topology Construction in the TOWN Project. TIK report 271, Computer Engineering and Networks Laboratory, ETH Zurich, March 2007. Available: http://www.tik.ethz.ch (see section: Publications)

[9] P. Cerutti, U. Fiedler, and R.Kohlas, Implementing a Protocol for Maintenance and Construction of Network Topologies in TOWN . Technical report, August 2007 (revised version). Available: http://www.tik.ethz.ch (see section: Publications)

[10] ITU-T Recommendation G.703, Series G: Transmission Systems and Media, Digital Systems and Networks, Digital terminal equipment - General, Physical/electrical characteristics of hierarchical digital interfaces. Nov. 2001

[11] ITU-T Recommendation G.704, Series G: Transmission Systems and Media, Digital Systems and Networks,Digital transmission systems-Terminal equipments - General, Synchronous frame structures used at 1544, 6312, 2048, 8448 and 44736 kbit/s hierarchical levels. Oct. 1998

[12] J. Moy, OSPF Version 2. RFC 2328, IETF, April 1998. 